\title{
A comparison of two exact methods for passenger railway rolling stock (re)scheduling
}

Haahr, Jørgen Thorlund; Wagenaar, Joris C.; Veelenturf, Lucas P.; Kroon, Leo G.

Published in:

Transportation Research. Part E: Logistics and Transportation Review

Link to article, DOI:

10.1016/j.tre.2016.03.019

Publication date:

2016

Document Version

Peer reviewed version

Link back to DTU Orbit

Citation (APA):

Haahr, J. T., Wagenaar, J. C., Veelenturf, L. P., \& Kroon, L. G. (2016). A comparison of two exact methods for passenger railway rolling stock (re)scheduling. Transportation Research. Part E: Logistics and Transportation Review, 91, 15-32. https://doi.org/10.1016/j.tre.2016.03.019

\section{General rights}

Copyright and moral rights for the publications made accessible in the public portal are retained by the authors and/or other copyright owners and it is a condition of accessing publications that users recognise and abide by the legal requirements associated with these rights.

- Users may download and print one copy of any publication from the public portal for the purpose of private study or research.

- You may not further distribute the material or use it for any profit-making activity or commercial gain

- You may freely distribute the URL identifying the publication in the public portal 


\title{
A Comparison of Two Exact Methods for Passenger Railway Rolling Stock (Re)Scheduling
}

\author{
Jørgen T. Haahr, Joris C. Wagenaar, Lucas P. Veelenturf, Leo G. Kroon
}

July 6, 2015

\section{Introduction}

In passenger railway optimization one of the main goals is to make efficient plans that can accommodate all passengers or, if that is not possible, that minimize the seat shortages. In the planning process, a railway operator tries to match the demand by first selecting an appropriate timetable followed by a matching rolling stock schedule. We focus on the second stage where a rolling stock schedule must be found given the timetable and passenger demand in this research. Not only the planning process, in which a long time ahead a rolling stock schedule must be determined, is considered, but also the real time construction of rolling stock schedules during disruptions.

If during operations an unexpected event causes the timetable, the rolling stock, and crew schedule to become infeasible, then these schedules need to be rescheduled. To ensure that the operations are not deadlocked, new feasible schedules must be found promptly. For a general overview of models for railway rescheduling during disruptions and disturbances we refer the reader to Cacchiani et al. [7, and for an overview on disruption management processes in general we refer to Jespersen-Groth et al. [16]. The major difference between rolling stock scheduling in the planning phase and rolling stock rescheduling during disruptions is the time available to come up with a solution. Next to that, during the rescheduling phase there is less flexibility since the trains are already running, and choices made before the disruption occurred cannot be reversed. A natural consequence is that it may not be possible to assign rolling stock to all train services, i.e., some train services may need to be cancelled. Therefore in the rescheduling case it is highly undesirable, but considered feasible, to assign no rolling stock to some services.

There is a body of literature tackling the problem of assigning rolling stock to passenger train services. These papers focus either on the planning process or on the rescheduling process. The majority of the approaches existing in literature considers one specific (and national) network, and does not benchmark against other approaches. In this paper we want to make a start with comparing 
different approaches, as well as comparing them on different networks. We hope to encourage other researchers to do the same.

Two main categories of rolling stock problems have been studied for passenger railways in literature. The first consists of assigning both carriages and locomotives to trips. Each carriage can be coupled individually and independently to a convoy (or composition), but at least one locomotive is required to pull the convoy. The other branch of research consists of assigning self-propelled train units that need not be pulled by a locomotive. These units consist of a fixed number of carriages and have their own traction engines. It is common that these train units can be coupled together to form larger train compositions.

The problem of assigning locomotives and/or carriages to trains can be applied to passenger trains (see e.g. Cordeau et al. [10, Cordeau et al. 11, Lingaya et al. [18, and Brucker et al. 6]), but also to freight trains (see e.g. Ahuja et al. [1, Rouillon et al. [21, Vaidyanathan et al. [22], and Bouzaiene-Ayari et al. [5]). The problem of (re)scheduling self-propelled units is also considered in multiple publications (see e.g. Alfieri et al. [3, Fioole et al. 13, Peeters and Kroon [20, Cadarso and Marín [8, Nielsen et al. [19], Cadarso and Marín [9], Borndörfer et al. 4, and Haahr et al. 14]). For more references, with respect to papers considering rescheduling approaches, we refer the interested reader to Cacchiani et al. [7].

In this paper we consider two rolling stock (re)scheduling approaches for self-propelled train units. The first approach is based on the algorithm introduced by Fioole et al. 13. This approach makes use of a general purpose solver (CPLEX) to solve a Mixed Integer Linear Program (MILP) for rolling stock (re)scheduling. The second approach is an extension of the algorithm introduced by Haahr et al. 14. This algorithm makes use of column generation to solve a MILP. We note that Peeters and Kroon 20 also investigated a column generation solution method for the rolling stock (re)scheduling problem. However, a different decomposition method was performed that did not involve path generation for individual rolling stock units.

The method of Haahr et al. [14] does not consider the order of rolling stock units within the train compositions. However, the order is of significant importance to determine which units can be decoupled from a composition. For example, at certain stations only the unit at the rear of a composition can be decoupled. Therefore we introduce a new formulation which considers the order of rolling stock units within compositions. This path based formulation requires adaptations to the column generation heuristic introduced by Haahr et al. [14]. To improve the performance in terms of computation time, a row generation variant of the algorithm is introduced as well.

The advantage of the path based formulation based on Haahr et al. [14] is that it models rolling stock unit duties (a list of subsequent trips) explicitly, thereby enabling dealing with unit specific constraints naturally; a good example of such constraints are maintenance appointments at workshops. An optimal solution of the MILP formulation of Fioole et al. [13] only contains information on which compositions are assigned to which trips and how compositions change between trips. However, it does not produce paths for each individual rolling 
stock unit. In a post processing step, a simple heuristic can construct these individual routes, because an integer flow can always be decomposed into unit valued path flows (see Ahuja et al. [2]). However, taking constraints on individual units into account is not possible. For a discussion and other solutions for this problem we refer the reader to Wagenaar et al. [23].

Although the Mixed Integer Linear Programs of the two approaches are different, they are set up such that they solve exactly the same problem. Since both solution methods are exact solution methods, they will come to solutions with the same objective value. Note that the solutions can differ if multiple solutions with the same objective value exist.

We benchmark both approaches on rolling stock scheduling and rescheduling instances of Netherlands Railways (NS) and the Copenhagen Suburban Railway Operator DSB S-tog. In the scheduling instances a timetable and the passenger demand are given. The methods must assign rolling stock compositions to every train service such that the seat coverage of passenger demand is satisfactory while minimizing operational costs.

For the rescheduling instances, the original rolling stock schedule, an updated timetable, and the passenger demand are given. In this paper we assume that the passenger demand will not change due to a disruption - modelling passenger demand during a disruption is out of the scope of this paper. In these instances the main objective is to assign an appropriate rolling stock composition to as many train services as possible. However, it is no longer a hard constraint that all train services need a rolling stock composition assigned to them. The secondary objectives are to cover the passenger demand as well as possible and to deviate as little as possible from the original schedule. All deviations from the original schedule require additional shunting movements like couplings and uncouplings of train units. Unplanned or cancelled shunting movements require additional communication and coordination with shunting personnel; in some cases even additional shunting personnel must be arranged. Introducing deviations to the planned schedule is thus not preferred, especially since the available time available is limited.

The remainder of this paper has the following structure. First, the contributions of this paper are discussed in Section 1.1. The problem description and assumptions are discussed in Section 2. The mathematical formulations for the two solution approaches are discussed in Section 3. In Section 4 computational experiments are presented. The paper is concluded in Section 5 .

\subsection{Contribution}

This paper has several methodological and practical contributions. The methodological contributions are the introduction of: i) a new mathematical formulation, ii) a new column generation approach, and iii) a new row generation approach. The new mathematical formulation is a path based MILP formulation for the rolling stock (re)scheduling problem. In this formulation the order of units within compositions is taken into account. The column generation approach is an extended version of the approach of Haahr et al. 14. The extension 
was necessary to incorporate the order of units within compositions. Finally, a row generation is adopted for a significant speedup in runtime of the solutions approach.

The practical contributions are: i) realistic tests on DSB S-tog and Netherlands Railways instances, $i i$ ) a comparison between different rolling stock (re)scheduling approaches, and iii) comparisons of different instances within different countries. Furthermore, we encourage other railway operations researchers to perform benchmarks as well. This is the first work that actually incorporates the order of units within compositions in test instances of DSB S-tog. The transition (i.e. composition) rules have been made in co-operation with DSB S-tog. A comparison is made between different rolling stock (re)scheduling approaches by testing them on the same data sets, and these approaches are benchmarked on two different railway networks, namely a large train service network in the Netherlands and the suburban network in Copenhagen, Denmark. Finally, we encourage other researchers to make such performance comparisons as well. This is of importance to bridge the gap between theory and practice.

\section{Problem description}

In this section we describe the rolling stock (re)scheduling problem in more detail, and the assumptions we make.

The rolling stock scheduling problem consists of assigning a rolling stock composition to every trip in the timetable of one planning horizon, e.g., one day of operation. The rolling stock rescheduling problem consists of assigning rolling stock compositions to as many trips as possible for the remainder of the day in case of a disruption. A trip is a part of a train service, as specified by the timetable, between two major stations where the composition of the train can be changed. A trip consists of a departure station, departure time, arrival station, and arrival time. Furthermore, a composition is an ordered set of coupled rolling stock units. The assignment of rolling stock compositions should be done such that it minimizes the number of seat shortages for passengers, the total number of carriage kilometers, and several other objectives. Consequently, the rolling stock (re)scheduling problem essentially represents a multi-criteria decision problem.

For each trip a maximum length of the allowed composition is given to ensure that the length of the composition assigned to a trip is not longer than the length of the shortest platform amongst all platforms where the train has a stop. Platform lengths along the network may differ, so different trips may have different maximum composition lengths.

After a trip has been operated the composition is usually assigned to a next succeeding trip. Such a combination of two succeeding trips is also called a connection (note that this also holds for end stations of a line). It is allowed to first change the composition before it is used on the next trip. However, the transition from one composition to another must follow certain business rules. Depending on the direction in which the trip is operated and the station layout, 


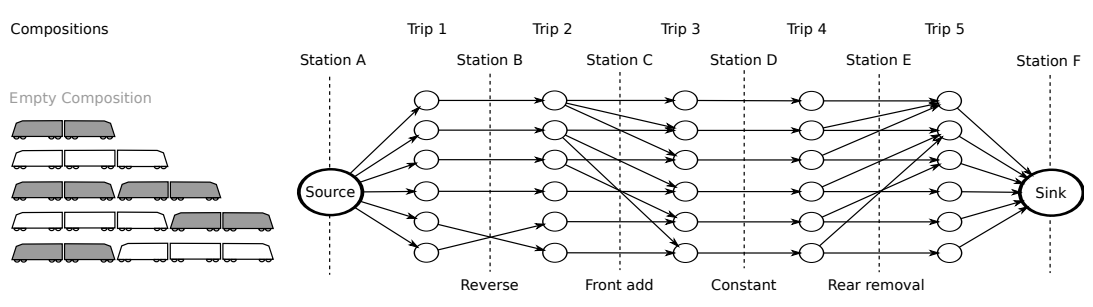

Figure 1: An example of a transition network illustrating how different rules affect the possible composition transitions from one trip to the successor trip. On the left the example compositions are represented, and to the right the transition network is depicted. Any path from the source to the sink represents a transition scheme that is feasible. A train travels from one station to the next. The transition rules depend on the station layout and business rules. Examples in the figure are: reversing the composition, only coupling units to the front, allowing no change, and only allowing units to be decoupled from the rear.

it is stated on which side of the composition it is allowed to add (couple) extra rolling stock units and on which side it is allowed to decouple units. Such a composition change is called a transition.

The possibility of coupling and decoupling units requires that we keep track of the order of the rolling stock units within the composition. Most of the time it is only allowed to (de)couple on one side of the composition. For example, in the Netherlands, if a train continues in the same direction, coupling is usually done at the front of the train. This will speed up the process since the rolling stock unit could, in this situation, already be placed there before the train arrives. Decoupling will most likely take place at the rear of the train if the train continues in the same direction. As a consequence, the train can leave before the decoupled unit is shunted away. Figure 1 shows an example of a transition network illustrating how different composition transition rules work. An explanation of the Figure is given in the caption. Keeping track of the order of the units within a composition makes the model more complicated than a simple assignment problem. Furthermore, coupling or decoupling units requires shunting personnel and time, and is therefore penalized in the objective function.

In this paper, we assume that shunting yards associated with certain stations have infinite capacity to accommodate all composition changes and to park decoupled rolling stock units. After decoupling a unit, we require a certain minimum time duration before that unit can be coupled to a new trip in another composition. This restriction reflects the time required to move a decoupled unit to the shunting yard and from the yard back to a platform to be coupled again.

For each trip an estimated passenger demand is given. It is not strictly required that the capacity for passengers of the composition assigned to a trip is equal or larger than the passenger demand for that trip. However, assigning a composition to a trip which cannot accommodate all passengers is penalized.

On the other hand, considering depreciation and energy efficiency, having 
more capacity than there is demand for a trip is also not preferred. Therefore, the number of kilometers driven by all carriages is penalized as well. A trade-off between the number of seat-shortages and the number of carriage kilometers must be found in the rolling stock circulation.

We must ensure that the next day the rolling stock schedule can be applied as planned. Therefore, the schedule must be such that at the end of the day the rolling stock units are spread over the shunting yards such that at each shunting yard there are as many rolling stock units as are required at the start of the next day (also called end-of-day balance). We allow differences in the end-of-day balance, but against a certain penalty, since for each negative unit difference a new deadhead trip should be planned during the night to re-balance the inventories. Deadheading is expensive in practice since it requires additional manpower and causes additional equipment depreciation.

Especially in the case of rescheduling, it is of significant importance that solutions are found very fast. The trains need to keep running, which means that the operator can not wait for one hour to decide how to adapt the resource schedules in order to handle the ongoing disruption.

\section{Mathematical formulations}

In this section we introduce the mathematical formulations of the two solution methods. The first formulation is the one of Nielsen et al. 19] (based on Fioole et al. 13]), also called the Composition Model and the second formulation is a path based (column generation) formulation based on Haahr et al. [14.

We will first introduce the common parts used in both formulations. Afterwards, in Sections 3.1 and 3.2 the model specific constraints are discussed.

In Tables 1,2 and 3 , the sets, parameters, and variables are introduced and explained respectively.

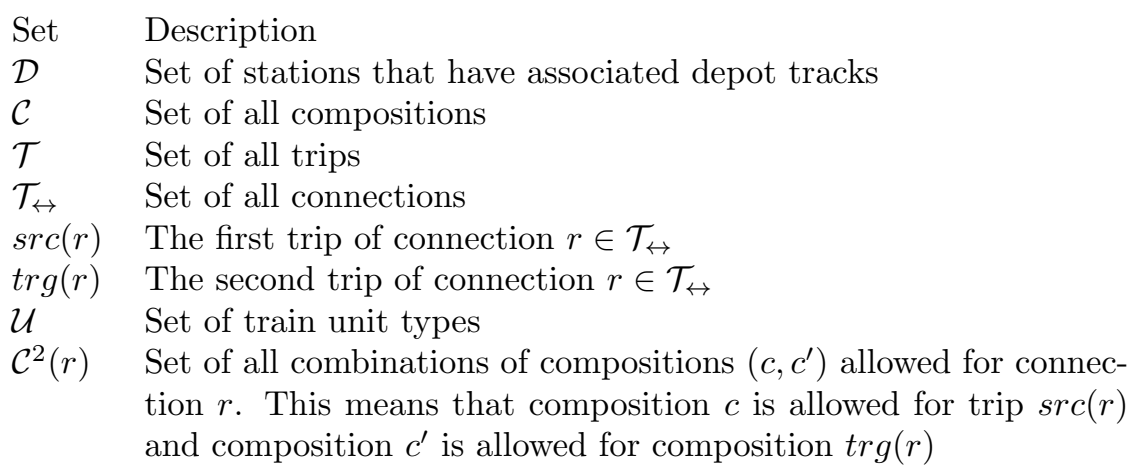

Table 1: List of sets and elements

The objective function can now be expressed in terms of the variables and 


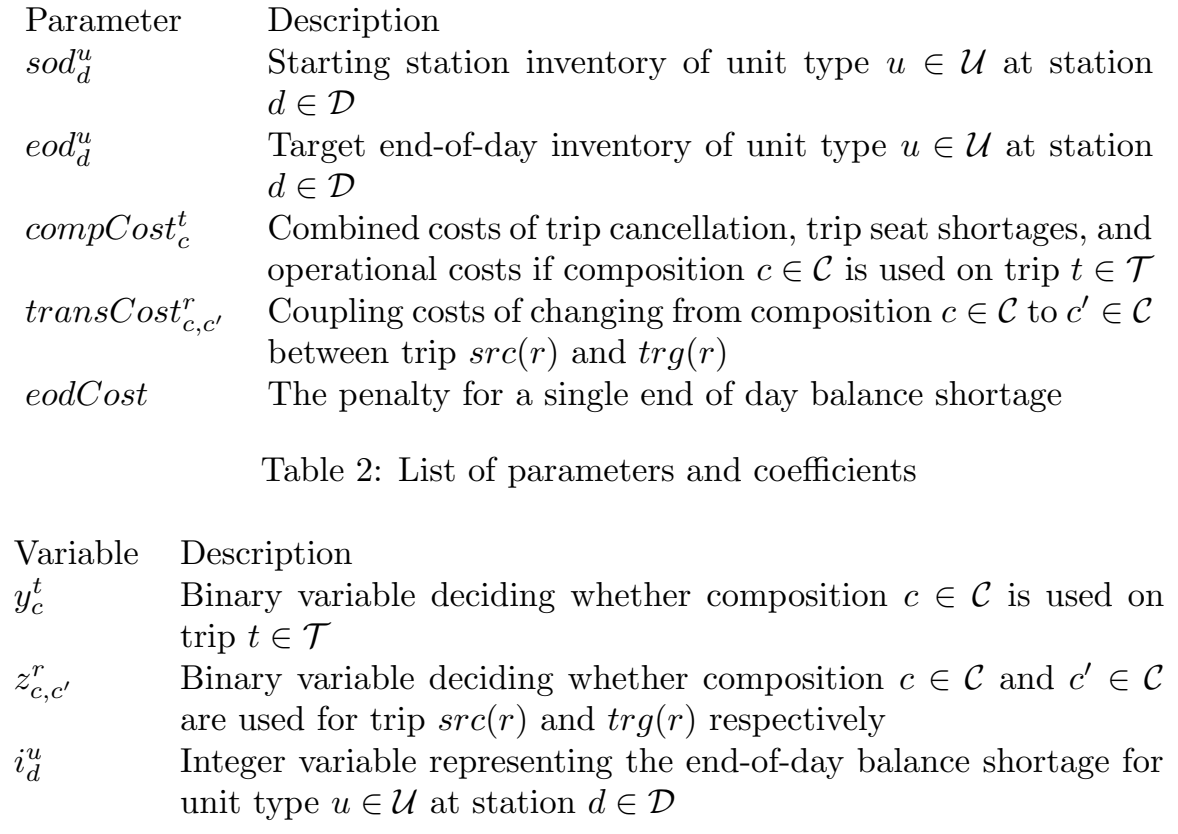

Table 2: List of parameters and coefficients

Variable Description

$y_{c}^{t} \quad$ Binary variable deciding whether composition $c \in \mathcal{C}$ is used on trip $t \in \mathcal{T}$

$z_{c, c^{\prime}}^{r} \quad$ Binary variable deciding whether composition $c \in \mathcal{C}$ and $c^{\prime} \in \mathcal{C}$ are used for trip $\operatorname{src}(r)$ and $\operatorname{trg}(r)$ respectively

$i_{d}^{u} \quad$ Integer variable representing the end-of-day balance shortage for unit type $u \in \mathcal{U}$ at station $d \in \mathcal{D}$

Table 3: List of Variables

coefficients:

$$
\begin{aligned}
\text { Minimize: } & \sum_{t \in \mathcal{T}} \sum_{c \in \mathcal{C}} \operatorname{compCost}_{c}^{t} \cdot y_{c}^{t} \\
& +\sum_{r \in \mathcal{T}_{\leftrightarrow}} \sum_{c \in \mathcal{C}} \sum_{c^{\prime} \in \mathcal{C}} \operatorname{trans}_{\operatorname{Cost}}^{r}, c^{\prime} \\
& +\sum_{u \in \mathcal{U}} \sum_{d \in \mathcal{D}} \operatorname{eodCost} \cdot i_{d}^{u}
\end{aligned}
$$

The objective function consists of three parts: costs for assigning compositions, costs for assigning transitions between compositions, and costs for having end of day balance shortages. Trip cancellation, seat shortage and operational costs are included in (1). The shunting costs are included in (2), and the end-of-day shortage costs are accounted for in (3).

Furthermore, both formulations will make use of the following constraints 
to assign compositions to the trips:

$$
\begin{array}{rlr}
\sum_{c \in \mathcal{C}} y_{c}^{t}=1 & \forall t \in \mathcal{T} \\
y_{c}^{s r c(r)}=\sum_{c^{\prime} \in \mathcal{C}:\left(c, c^{\prime}\right) \in \mathcal{C}^{2}(r)} z_{c, c^{\prime}}^{r} & \forall r \in \mathcal{T}_{\leftrightarrow}, c \in \mathcal{C} \\
y_{c^{\prime}}^{\operatorname{trg}(r)}=\sum_{c \in \mathcal{C}:\left(c, c^{\prime}\right) \in \mathcal{C}^{2}(r)} z_{c, c^{\prime}}^{r} & \forall r \in \mathcal{T}_{\leftrightarrow}, c^{\prime} \in \mathcal{C}
\end{array}
$$

Constraints (4) ensure that to each trip exactly one composition is appointed and Constraints (5) and (6) ensure that a feasible path is found in the transition network, see Figure 1. The composition appointed to the incoming trip in a connection $\left(y_{c}^{s r c(r)}\right)$ must be equal to the actual incoming composition in the chosen transition, as modelled by Constraints (5). On the other hand, the composition appointed to the outgoing trip in a connection $\left(y_{c^{\prime}}^{\operatorname{trg}(r)}\right)$ must be equal to the actual outgoing composition in the chosen transition, as modelled by Constraints $(6)$.

These constraints do not consider the availability of rolling stock units and do not measure the end-of-day shortages $i_{d}^{u}$. In the following two sections we discuss how the two different models take this into account.

\subsection{The Composition Model}

The first option to consider the availability of rolling stock is to keep track of the inventory of rolling stock unit types at the stations. This option is applied in the formulation of Nielsen et al. [19], which is based on Fioole et al. [13. In this section this formulation is summarized. Note that some of the notation is changed to align it with the model presented in Section 3.2.

For this formulation additional parameters and variables are necessary to determine at each station the inventory and the number of coupled and decoupled units. The parameters $\operatorname{coup}_{c, c^{\prime}}^{u}$ and uncoup ${\text { u }, c^{\prime}}^{u}$ indicate how many rolling stock units of type $u \in \mathcal{U}$ should be coupled or uncoupled respectively if the composition changes from $c \in \mathcal{C}$ to $c^{\prime} \in \mathcal{C}$. These values can not be negative. For instance, if 2 units of type $u$ need to be coupled during a composition change from composition $c$ to composition $c^{\prime}$, then $\operatorname{coup}_{c, c^{\prime}}^{u}=2$ and uncoup $p_{c, c^{\prime}}^{u}=0$. Furthermore, we assume a certain processing time to shunt a decoupled unit to a shunting yard and to get it back from the shunting yard. Therefore, a parameter $\tau_{r}^{-}$is used for each connection $r$ indicating the time it will take before the units that are decoupled during connection $r \in \mathcal{T}_{\leftrightarrow}$ (in between trips $\operatorname{src}(r)$ and $\operatorname{trg}(r))$ are available again for coupling. Also a parameter $\tau_{r}^{+}$is required for each connection $r$ indicating the time at which connection $r$ takes place. This is the time the units which need to be coupled to the composition between trips $\operatorname{src}(r)$ and $\operatorname{trg}(r)$ should be available. The station at which connection $r$ takes place is indicated by $d(r)$. Note that this is the station where trip $\operatorname{src}(r)$ ends and where trip $\operatorname{trg}(r)$ starts. 
For connection $r \in \mathcal{T}_{\leftrightarrow}$ and rolling stock unit type $u \in \mathcal{U}$, the non-negative integer variables $v_{r, u}^{+}$and $v_{r, u}^{-}$indicate respectively the number of rolling stock units of type $u$ that are coupled to the composition between trips $\operatorname{src}(r)$ and $\operatorname{trg}(r)$, and the number of rolling stock units of type $u$ that are uncoupled from the composition between trips $\operatorname{src}(r)$ and $\operatorname{trg}(r)$. Furthermore, a variable $i n v_{r, u}$, representing the inventory just after connection $r \in \mathcal{T}_{\leftrightarrow}\left(\tau_{t}^{+}\right)$of rolling stock units of type $u \in \mathcal{U}$ at station $d(r)$, is required.

Then the following constraints are added to ensure that: i) the inventory is non negative at each station and each time period and ii) the end-of-day balance shortage is correctly measured.

$$
\begin{aligned}
& v_{r, u}^{+}=\sum_{c \in \mathcal{C}} \sum_{\substack{c^{\prime} \in \mathcal{C}: \\
\left(c, c^{\prime}\right) \in \mathcal{C}^{2}(r)}} \operatorname{coup}_{c, c^{\prime}}^{u} z_{c, c^{\prime}}^{r} \\
& v_{r, u}^{-}=\sum_{c \in \mathcal{C}} \sum_{\substack{c^{\prime} \in \mathcal{C}: \\
\left(c, c^{\prime}\right) \in \mathcal{C}^{2}(r)}} \operatorname{uncoup}_{c, c^{\prime}}^{u} z_{c, c^{\prime}}^{r} \\
& i n v_{r, u}=\operatorname{sod}_{d(r)}^{u}-\sum_{\begin{array}{c}
r^{\prime} \in \mathcal{T}_{\leftrightarrow:}: \\
\left(r^{\prime}\right)=d(r), \\
\tau^{\prime} \leq \tau_{r}^{+}
\end{array}} v_{r^{\prime}, u}^{+}+\sum_{\begin{array}{r}
r^{\prime} \in \mathcal{T}_{\leftrightarrow}: \\
d\left(r^{\prime}\right)=d(r), \\
\tau_{r^{\prime}} \leq \tau_{r}^{+}
\end{array}} v_{r^{\prime}, u}^{-} \\
& i_{d}^{u} \geq\left(\operatorname{sod}_{d(r)}^{u}-\operatorname{eod}_{d}^{u}\right)-\left(\sum_{r \in \mathcal{T}_{\leftrightarrow}: d(r)=d} v_{r^{\prime}, u}^{+}-\sum_{r \in \mathcal{T}_{\leftrightarrow}: d(r)=d} v_{r^{\prime}, u}^{-}\right) \\
& i_{d}^{u} \in \mathbb{Z}^{+} \\
& v_{r, u}^{+}, v_{r, u}^{-}, i n v_{r, u} \in \mathbb{R}^{+}
\end{aligned}
$$

With this information Constraints (9) determine the inventory just after a connection takes place. This inventory is equal to the start inventory at the station, minus all units being coupled to compositions in earlier connections, plus all units which are available again after being uncoupled from compositions in earlier connections. Note, that since the variables $i n v_{r, u}$ are non-negative it also ensures that the inventory is zero or positive at all times. Constraints (10) measure at each station the number of units short at the end of the day with respect to the target end-of-day inventory. This is equal to the planned difference between the start and end inventory, minus the realized difference between the number of units coupled (departed) and uncoupled (arrived) during the day at that station. Constraints (11) and 12 ensure the non-negativity 
of the number of (un)couplings, inventories and end-of-day shortages. We note that the integrality of the end-of-day balance variables can be relaxed as these will be naturally integer valued.

\subsection{The Path Based Model}

In Section 3.1 a flow based model for the Rolling Stock (Re)Scheduling Problem is presented that identifies the correct number of units flowing from station to station. The Rolling Stock (Re)scheduling Problem can also be formulated using a path based model. In contrast to the flow based model, the path based formulation explicitly considers the route of each individual rolling stock unit and in this way availability of rolling stock is guaranteed. Although not considered in this paper, the path based formulation provides some advantages in terms of modelling unit specific constraints such as a maximal kilometer usage before maintenance, or enforcing a unit to meet a maintenance appointment.

The number of possible paths for a rolling stock unit grows exponentially by the number of trips, making the full path model computationally intractable. We therefore propose to solve this method by using column generation, i.e., only a subset of all possible paths is considered. By iteratively solving this reduced model (also known as the master problem) and adding columns with negative reduced cost (found using a so-called subproblem) we are able to get an optimal solution. We refer to Desaulniers et al. 12 for a detailed introduction to column generation and Branch and Price frameworks.

Recall from Section 1 that this model is an extension of the model presented in Haahr et al. 14]. The model is solved using a Branch and Price framework, i.e, a Branch And Bound (BAB) approach where columns are added dynamically (as needed to prove optimality) at every node in the BAB search tree. The main difference is that we have to consider the order of units within a composition. Therefore Constraints (4)-(6) are used in this formulation as well. We note that there are two alternative formulations with respect to the composition transition rules (Constraints (5)-(6)). First of all, they can be replaced by pure constraints on the pairs of trips in $\left(\mathcal{T}_{\leftrightarrow}\right)$. Preliminary results however show that this weakens the LP relaxation too drastically. The LP program becomes smaller and therefore also faster, however the relaxed solutions are more fractional. Another solution is to replace them with path conflict pair (or clique) constraints, i.e., forbid any pair that violates a transition rule. This approach was not tested as it would require significant changes to the master and subproblem. Each potential conflict constraint would need to be present in the master problem incurring a very large number of additional constraints. In this approach compositions variables are no longer necessary, as violation of the composition rules can be handled using the same conflict constraints. The subproblem would no longer be simple as it is non-trivial to account for the contribution to the new conflict constraints of a generated path. Two path are only in conflict if they form certain coupling and decoupling patterns which is hard to determine using a arc extension based shortest path method such as Dijkstra or a Label Setting Algorithm, see Irnich and Desaulniers [15]. 
In the remainder of this formulation, $\mathcal{P}_{d}^{u}$ is defined as the set of all paths for unit type $u \in \mathcal{U}$ starting in station $d \in \mathcal{D}$. A path describes a chronological list of trips that are performed by a single rolling stock unit, i.e., a unit's schedule for the planning period. The set of all possible paths is thus denoted by $\mathcal{P}:=$ $\bigcup_{u \in \mathcal{U}, d \in \mathcal{D}} \mathcal{P}_{d}^{u}$. Shunting operations are implicit as a unit has to be decoupled (and therefore shunted) whenever the unit exits a trip connection, and coupled whenever the unit, going out of the shunting yard, enters a connection.

The (re)scheduling problem simply consists of assigning exactly one path to each vehicle, subject to a number of constraints. Therefore, the master formulation contains additional sets of binary decision parameters and variables. The first set of binary parameters, $\alpha_{p}^{t}$, states for each path $p \in \mathcal{P}$ and trip $t \in \mathcal{T}$ whether path $p$ visits trip $t\left(\alpha_{p}^{t}=1\right)$ or not $\left(\alpha_{p}^{t}=0\right)$. The second set of binary parameters, $\beta_{p}^{d}$, takes the value 1 if path $p \in \mathcal{P}$ terminates at station $d \in \mathcal{D}$, and otherwise equals 0 . The binary variables are $\lambda_{p} \in\{0,1\}$ determine whether path $p \in \mathcal{P}$ is selected in the final solution or not.

We note that the penalties in (1) and (2) can be included in the subproblem instead of in the master problem, or alternatively partially included in both. Our preliminary results have shown that it is beneficial for the overall column generation convergence to put a part of the coupling costs in the subproblem. Without awareness of the coupling costs in the subproblem it may generate paths that are unnecessarily expensive, and possibly also incompatible in the master problem.

This leads to the following constraints in the master problem:

$$
\begin{array}{cc}
\text { Constraints: } & \forall u \in \mathcal{U}, t \in \mathcal{T} \\
\sum_{d \in D} \sum_{p \in \mathcal{P}_{d}^{u}} \alpha_{p}^{t} \lambda_{p}=\sum_{c \in \mathcal{C}} \mu_{t}^{u} y_{c}^{t} & \forall u \in \mathcal{U}, d \in \mathcal{D} \\
\sum_{p \in \mathcal{P}_{d}^{u}} \lambda_{p}=\operatorname{sod}_{d}^{u} & \forall u \in \mathcal{U}, d \in \mathcal{D} \\
i_{d}^{u}+\sum_{p \in \mathcal{P}_{d}^{u}} \beta_{p}^{d} \lambda_{p} \geq \operatorname{eod}_{d}^{u} & \\
\lambda_{p} \in\{0,1\}, \quad y_{c}^{t} \in\{0,1\}, \quad i_{d}^{u} \in \mathbb{Z}_{0}^{+} &
\end{array}
$$

Without equations (4)-(6) the master problem solely consists of finding a set of rolling stock paths. Together with Constraints $(13)$ these constraints ensure that only feasible compositions and composition transitions are made.

In comparison with Nielsen et al. [19] the path-formulation does not consider inventories at the stations, but it models individual paths for rolling stock units, thereby implicitly handling the inventories. The composition and path variables are linked by Constraints (13) to ensure that the correct number of rolling stock units (and types) are assigned to each trip composition. Constraints (14) ensure that exactly one path is assigned to every rolling stock unit. The endof-day balance is enforced in Constraints (15). A slack (which is penalized in the objective) is inflicted if insufficient units terminate at respective stations. 
Finally, the domains of the variables are shown in (16). We note that the integrality of the end-of-day balance variables can be relaxed as these will be naturally integer valued.

The master problem ((1)-(6) and (13)-(16) ) is solved iteratively while adding new columns (path variables) that have negative reduced cost. A subproblem is solved, using the duals of (13)- 15 , to find such columns or to prove that they do not exist. This problem can be solved as a shortest path problem (or with resource constraints when unit-specific constraints are enforced). When no columns exist with negative reduced cost we have solved the LP relaxation to optimality.

A Branch and Price framework is required to solve the problem, as the LP relaxation of the master problem is not necessarily integral. The framework setup and branching rules described by Haahr et al. [14 are adopted. A fractional solution can be broken by inserting constraints into the master problem that stipulate that the number of units on one trip arc is integral, and that the number of units originating or ending in a station is integral. In addition to the existing branching rules we also introduce branching on the composition variables. This type of branching partitions the transition variables associated to a connection into two groups. In each branch the sum of one group should be greater than or equal to one, while the other sum is zero.

\section{Subproblem Graph Example}

An example of the subproblem graph is shown in Figure 2, Note that the underlying graph is acyclic and the weights can be negative. We refer to Haahr et al. 14. for an in-depth description. Note, that the structure of the subproblem is the same as in Haahr et al. 14] even though we take compositions into account in the overall method. The example contains three stations whose events (departures and arrivals) are shown as vertically aligned vertices. Stations with associated depot tracks have a paired (gray color) node, representing the depot (i.e. shunting yard). The graph contains one source (S) that has one out-going arc to the first events of all stations, and one target node (T) that has one in-going arc for the last events on different stations. Intermediate arcs represent train movements (moving units between the depot tracks and a platform) and trips travelling from one station to another. Mileage costs are set on trip arcs and coupling costs are set on shunting arcs. Dual costs from the linking Constraints (13) are assigned to the trip arcs. Inventory duals from Constraints (14) are assigned to the arcs extending from the source. Finally, duals from Constraints (15), on the end-of-day balance, are assigned to the target node arcs. We note, that the subproblem can w.l.o.g. be changed slightly in implementation as some nodes and edges can be altered or removed.

\subsection{Delayed Transition Constraint Generation}

Preliminary results show that the LP-relaxation of the formulation requires

a significant amount of pivot operations to solve. This is highly undesirable 


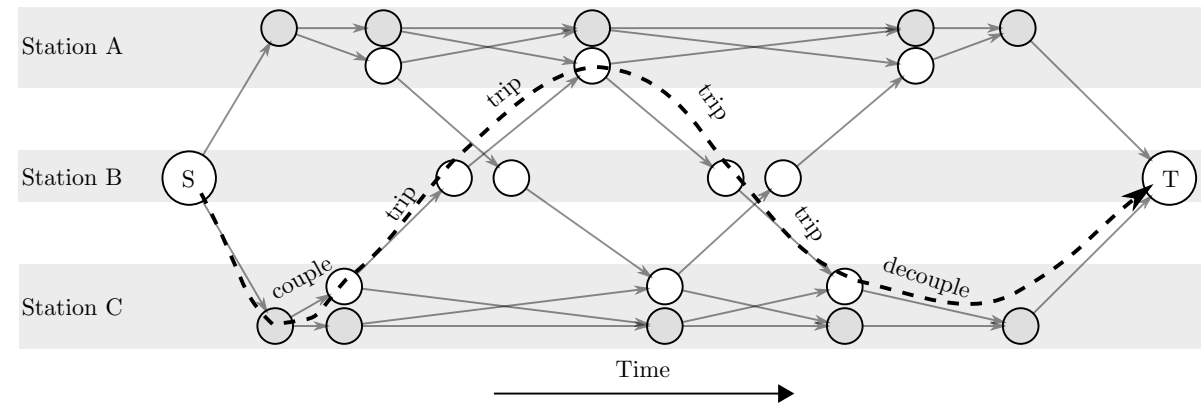

Figure 2: A simplified example of the acyclic time-space network showing some of the possible coupling arcs. Three stations (A,B and C) are illustrated where only two of them (A and C) have associated depot tracks. An example of a feasible unit schedule has been highlighted using a dashed path.

since we are continuously solving the formulation after adding columns in every iteration of the column generation process. We discovered that solving the linear relaxation of the master problem with the Dual Simplex Method was significantly faster than using the Primal Simplex Method. In column generation we unfortunately can not use the Dual Simplex Method efficiently since adding new columns may leave the current basis solution infeasible.

These observations did however lead us to experiment with the formulation in order to improve convergence or runtime speed of the linear relaxation. Constraints (5) and (6) not only represent a significant amount of constraints in the presented formulation, but also demand a rather restricted set of columns in order to remain feasible during the whole process. As a result, unnecessary columns are generated making the approach slow. When converging against the optimal linear relaxation, these constraints are unnecessarily restrictive as the intermediate set of states (fractional LP bases) is not important. By removing these constraints from consideration initially, the advantages are three-fold: we achieve a smaller linear program to resolve, we may gain a faster convergence, and finally we get better duals due to the omission of equality constraints. However, this extension comes at the cost of more added complexity. In the method we therefore propose a variant that dynamically adds Constraints (5) and (6) as they become violated once the linear program has converged. This step merely consists of looping through the allowed composition transitions and checking whether the current solution violates any constraint.

For the sake of simplicity we only add the dynamic constraints once no negative reduced cost columns can be found anymore. Thus, the proposed variant searches for new columns first, and for violated constraints second. Note, when adding new constraints the framework searches for negative reduced cost columns again since a new constraint changes the solution. We have solved the LP model to optimality once no columns can be generated and none of the constraints are violated. 


\section{Computational Experiments}

In this section we present and discuss our computational experiments. We test both models on a scheduling case from Netherlands Railways (NS) and on a scheduling case from the Copenhagen Suburban Railway Operator (DSB S-tog). Additionally, we test the models on different rescheduling cases from both NS and DSB S-tog. In this way we thus make a comparison between the (re)scheduling approaches.

We start with describing the rolling stock scheduling instances of both networks and introduce the parameter settings we have adopted. These parameter settings are chosen after several runs to benchmark their influence. Thereafter, a comparison of the computational results of the scheduling instances is made. Then, the rescheduling instances are described in detail for both the NS and DSB S-tog instances. Finally, we conclude the section with an overview of the different rescheduling results.

\subsection{Rolling Stock Scheduling}

\subsubsection{Netherlands Railways}

A single instance on the Dutch railway network that spans the major part of the intercity network of NS is selected as computational case. Figure 3 shows the trajectory of the network. The majority of the lines are in the western part of the Netherlands which is the busiest part of the Dutch railway network. In total we consider the timetable of 16 distinct lines of a certain weekday, namely the Monday. There are different timetables required on the Friday and in the weekend. However, the differences between those timetables are small, while the differences in passenger demand could be large. Therefore, we only consider the timetable of the Monday as scheduling instance. In general the lines are operated half-hourly, but some are operated on an hourly basis. This leads to a total of 2324 trips between 14 different major stations. After preprocessing the total number of non-reducible trips is 727 , i.e., in fact only 727 trip compositions need to be decided as the rest will be fixed due to the composition transition rules.

We have two different rolling stock types available. A rolling stock unit of type $a$ consists of 4 carriages and has a passenger capacity of 405 seats, and a rolling stock unit of type $b$ consists of 6 carriages and has a passenger capacity of 597 seats. The maximum composition length for all trips is 14 carriages in the considered network. This leads in total to 11 different compositions that can be appointed to a trip: $a, a a, a a a, b, b b, a b, b a, a a b, a b a, b a a$, and the empty composition meaning that a trip is cancelled.

In the scheduling phase of NS the goal is to determine the start and end inventory at every station, and to appoint compositions to trips to fulfill the passenger demand. This is solved in two steps. First a suitable initial fleet distribution is found. Afterwards the found initial inventory setting is used to create the rolling stock circulation. 


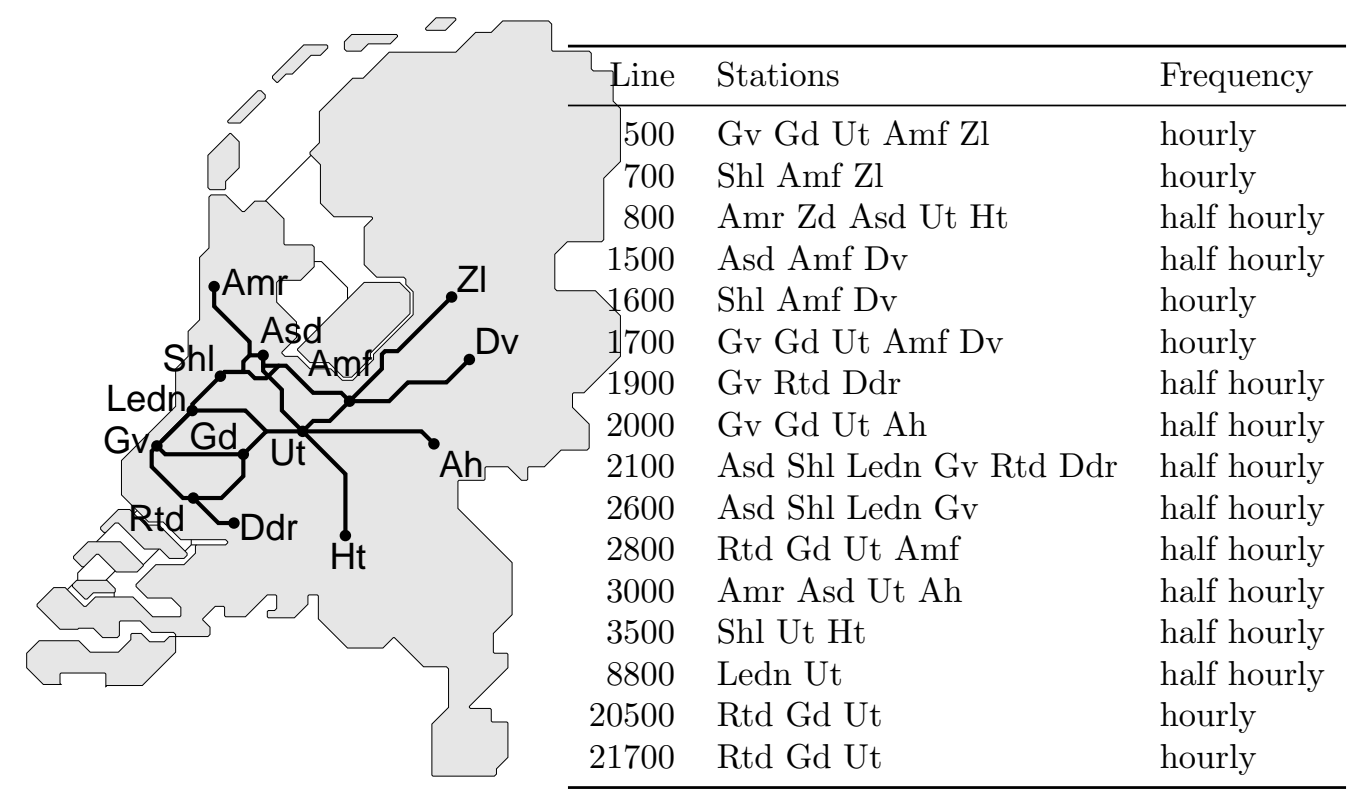

Figure 3: The NS network considered in the test instances.

In the first step, our objective is to create a rolling stock circulation that covers all demand while using the least amount of carriages. To this end, the model determines the number of rolling stock units required at the start of the day at each station to create such a circulation. We set a small penalty on the number of carriage kilometers. No penalty at all is set on the number of shunting operations applied during the day, and no penalty is given for deviations between the start and end inventory.

Our first step provides us with a start circulation that contains in total 113 units of type $a$ and 58 units of type $b$. This start circulation is able to cover all passenger demand.

In the second step, we use the start inventory created in the first step as fixed input. It is not allowed to deviate from this start inventory and the objective is then to find a rolling stock circulation that minimizes the costs using the coefficients as shown in Table 4. These penalty values are commonly used in literature, but we check the influence of changing the Mileage and Seat Shortage penalty ratio on the results in Section 4.1.3.

Here, Cancel means the penalty for cancelling a trip, Mileage denotes the penalty for driving a single carriage over one kilometer, Seat Shortage defines the penalty for having a seat shortage per passenger shortage per kilometer, Shunting refers to the penalty for doing a shunting operation, and, finally, Start end diff denotes the penalty for a negative difference between the start and end 
inventory at a station. Results are shown and discussed in Section 4.1.3.

\begin{tabular}{lr}
\hline Coefficient & NS \\
\hline Cancel & 1000000 \\
Mileage & 1 \\
Seat Shortage & 0.5 \\
Shunting & 1000 \\
Start end diff & 10000 \\
\hline
\end{tabular}

Table 4: Penalty values used for the NS scheduling case

\subsubsection{DSB S-tog}

The suburban train service network provided by DSB S-tog in Copenhagen is operated using a weekly periodic timetable and an hourly periodic timetable on a daily basis. Weekdays and weekends are operated using slightly different timetables, and an additional set of night trains are operated on Friday and Saturday nights. This leaves a total of four different timetable schedules: Monday (to Thursday), Friday, Saturday and Sunday. Table 5 summarizes the instance specifics. Note that the $A, B, C$, and $F$ lines are considered in all cases, while the Monday and Friday instances contain more lines than just these 4 .

\begin{tabular}{lrrrll}
\hline Name & Stops & Trips & Trips* & Weekday & Lines \\
\hline DSBfri & 28719 & 4558 & 886 & Friday & A,B,Bx,C,E,F\&H \\
DSBsat & 20474 & 1916 & 590 & Saturday & A,B,C\&F \\
DSBsun & 19919 & 1871 & 574 & Sunday & A,B,C\&F \\
DSBmon & 28017 & 4468 & 868 & Monday & A,B,Bx,C,E,F\&H \\
\hline
\end{tabular}

Table 5: Four timetables operated by DSB S-tog. The columns respectively show the instance names, total number of stops, total number of trips, total number of non-reducible trips (Trips*), weekday, and finally the lines that are running.

Two unit types are used to perform all train services. The smaller unit type $e$ is 42 meters long and contains 150 passenger seats, the larger unit type $f$ is 83 meters and holds 336 passenger seats. Platform lengths vary from station to station in Denmark as well, and the current lines partition the composition lengths into two sets. The first set allows only small compositions $e, e e$, and $f$. In addition to this, the seconds set also allows larger compositions eee, fe, ef, and $f f$.

We use distinct parameter settings for NS and S-tog since the characteristics of the network and train unit fleets are different. The parameter settings for the S-tog network can be found in Table 6. As can be seen, the only difference between the two settings is the mileage penalty. This is, among other factors, 


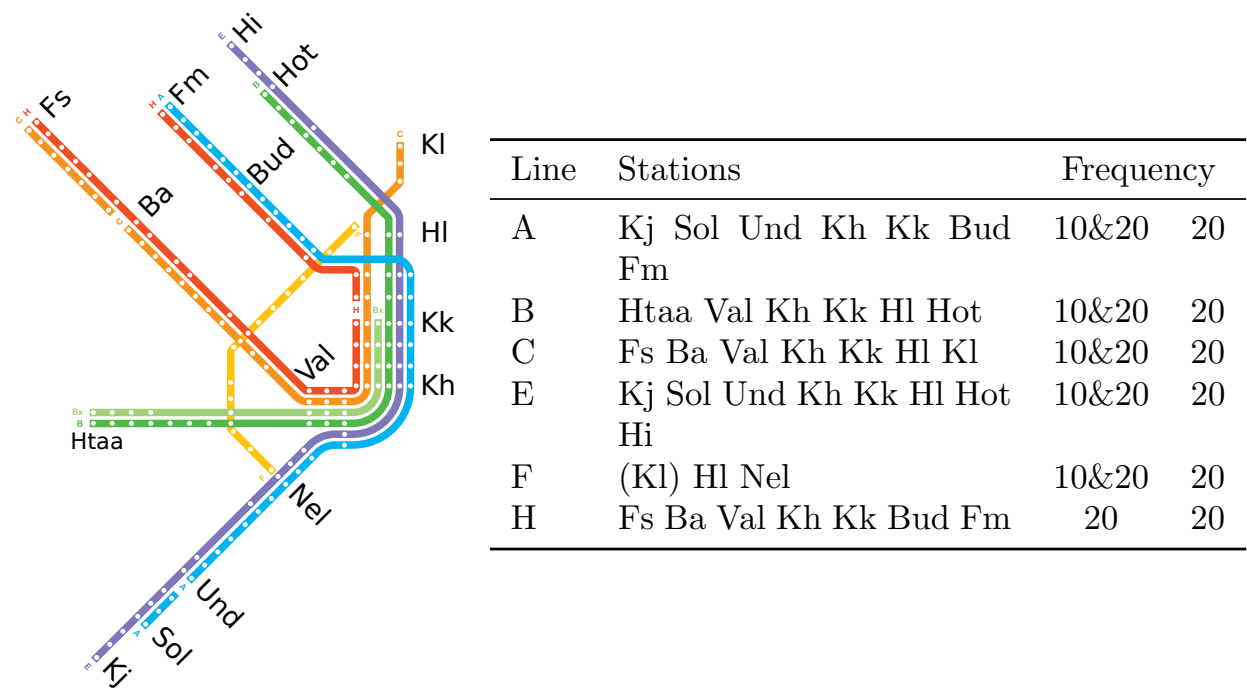

Figure 4: The DSB S-tog network considered in the test instances. Only key stations are listed in the table. The table columns show line name, station name and the operated frequencies in minutes for Monday-Friday and SaturdaySunday respectively.

because the rolling stock units of DSB S-tog are measured by length in meters in contrast to the number of carriages. The length in meters is substantially larger than the number of carriages.

\begin{tabular}{lr}
\hline Coefficient & DSB S-tog \\
\hline Cancel & 1000000 \\
Mileage & 0.1 \\
Seat Shortage & 0.5 \\
Shunting & 1000 \\
Start end diff & 10000 \\
\hline
\end{tabular}

Table 6: Penalty values used for the DSB S-tog scheduling case

DSB S-tog provided us with a start inventory. Thus, for S-tog instances it is not necessary to perform the first step of scheduling, that was described for NS instances. The start inventory contains 31 units of type $e$ and 103 units of type $f$.

\subsubsection{Computational results}

In the scheduling phase we determine the start inventory at every station, the end inventory at every station, and we appoint compositions to trips to transport 
passengers. For the NS case, the first phase, to create a start inventory, is solved within seconds and the start inventory is fixed from here on. Note that the end inventory is not fixed, but we penalize for deviations with respect to the start inventory, assuming that the next day the same schedule needs to be operated. Secondly, note that for the DSB S-tog instances we skip the first phase and adopt the provided initial inventory.

In this section we benchmark the two proposed methods for solving the rolling stock scheduling problem on both NS and DSB S-tog instances. We compare both NS and DSB S-tog instances on the second phase of scheduling. The mathematical formulations are equivalent and we have verified that the optimal objective costs are identical for the two methods. The objective function is a mix of several penalties where the exact balance between them is non-trivial to set. Due to this, there is little justification for solving to true optimality. In practice, it might be enough to accept solutions that are within $1 \%$ of optimality, but to be able to compare the approaches accurately we solve to optimality.

A study showing the trade-off between the seat shortage and mileage cost is first performed. The results are shown in Figure 5. The results show a steady increase in mileage costs as the seat cover moves towards 100\%. However, when we get close to $99 \%$ we observe diminishing returns. A cover of $100 \%$ is preferred by rolling stock planners, however not at all cost. In practice a seat cover close to $100 \%$ with a rolling stock circulation that is not overly expensive is chosen.

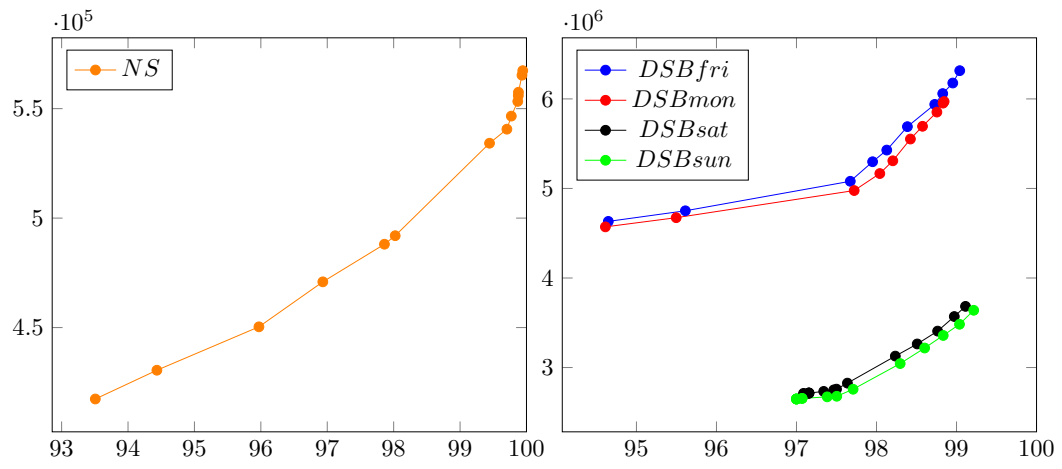

Figure 5: Trade-off between mileage and seat/demand cover for the NS (left) and DSB S-tog (right) instance. The vertical and horizontal axis respectively show the mileage usage (in $\mathrm{km}$ ) and seat cover percentage.

Next to the costs of the rolling stock circulation, the computation time to find the circulation is of importance as well. Information about how the computation times are affected by the different penalties for seat shortages are provided in Table 7 for all scheduling instances. Here $A$ denotes the Path Based Model without row generation, $B$ the Path Based Model with row generation, and $N$ the Composition Model. As can be seen, $B$ is able to solve the scheduling instances on average faster than $A$. This will be discussed in more detail in Section 4.3. Furthermore, the Composition Model is able to solve the instances 
even faster, but all solution times are acceptable for the planning phase. We assume that a solution method resulting in optimal schedules within 15 minutes can be used in practice.

\begin{tabular}{r|rrr|rrr|rrr|rrr|rrr|rr}
\hline & \multicolumn{4}{|c}{ NS } & \multicolumn{3}{|c|}{ DSBmon } & \multicolumn{3}{|c|}{ DSBfri } & \multicolumn{3}{|c}{ DSBsat } & \multicolumn{3}{c}{ DSBun } \\
Seat shortage costs & $A$ & $B$ & $N$ & $A$ & $B$ & $N$ & $A$ & $B$ & $N$ & $A$ & $B$ & $N$ & $A$ & $B$ & $N$ \\
\hline 0.1 & 444 & 84 & 27 & 398 & 30 & 14 & 314 & 63 & 14 & 14 & 3 & 2 & 21 & 3 & 3 \\
0.2 & 545 & 65 & 23 & 575 & 44 & 13 & 423 & 59 & 13 & 21 & 6 & 3 & 29 & 4 & 2 \\
0.3 & 742 & 274 & 16 & 652 & 345 & 21 & 556 & 48 & 21 & 31 & 6 & 3 & 40 & 4 & 3 \\
0.4 & 712 & 493 & 17 & 579 & 96 & 14 & 479 & 38 & 12 & 57 & 5 & 4 & 41 & 4 & 4 \\
0.5 & 709 & 465 & 19 & 653 & 119 & 19 & 664 & 37 & 12 & 58 & 10 & 7 & 35 & 4 & 2 \\
0.6 & 946 & 781 & 16 & 760 & 47 & 11 & 709 & 40 & 14 & 36 & 8 & 5 & 31 & 4 & 3 \\
\hline
\end{tabular}

Table 7: Detailed runtimes using the solution methods on the non-disrupted test instances. The columns respectively show the computation times to solve the instances with $A, B$, and $N$ method.

Recall that the Composition Model and the Path Based Model give the same results in terms of objective values. We have selected an appropriate balance in the following benchmarks by choosing the settings as shown in Table 4 and Table6. These settings lead to the results as shown in Table 8. We have left out the results of the $A$ method, because they are all dominated by the $B$ method. Note that in the DSB S-tog cases it is not possible to reach a seat coverage equal to $100 \%$. The seat coverage for all instances is above $98 \%$, so almost all passengers fit in the appointed compositions with a seat.

\begin{tabular}{lccccccccc}
\hline Instance & Obj & MC & SSC & SC & $\delta$ & Cover & $B(\mathrm{~s})$ & Columns & $N(\mathrm{~s})$ \\
\hline NS & 639065 & 553310 & 15755 & 70000 & 0 & $99.9 \%$ & 465 & 14340 & 19 \\
DSBmon & 719184 & 555970 & 132214 & 31000 & 0 & $98.5 \%$ & 119 & 5937 & 19 \\
DSBfri & 727159 & 583505 & 119654 & 24000 & 0 & $98.6 \%$ & 37 & 3627 & 12 \\
DSBsat & 418148 & 313469 & 87679 & 17000 & 0 & $98.3 \%$ & 10 & 1052 & 7 \\
DSBsun & 413062 & 297574 & 93489 & 22000 & 0 & $98.1 \%$ & 4 & 1266 & 2 \\
\hline
\end{tabular}

Table 8: Scheduling Results. The columns respectively show the solved instance, the objective value, the mileage kilometer costs, the overall seat-shortage kilometer costs, the costs for performing shunting operations, the costs for negative differences between start and end inventories, the total seat cover percentage, computation times and columns generated using the $B$ method, and finally the computation times for the $N$ method.

\subsection{Rolling Stock Rescheduling}

In this section we discuss the computational experiments for the rescheduling instances. Note that for the rescheduling instances we assume the demand to 
be the same as in the original situation. So, on the trips that have not been cancelled there is no changed demand due to the disruption. This means that as soon as a trip is cancelled, all passenger demand of that trip is assumed to vanish. See, for instance, Kroon et al. 17 for a paper that takes dynamic passenger flows into account by means of a simulation step in the Rolling Stock Rescheduling Problem. This aspect is outside the scope of the current paper. In this section, we first give a brief overview of the different rescheduling cases for Netherlands Railways (Section 4.2.1) and DSB S-tog (Section 4.2.2). Thereafter, the computational results are discussed (Section 4.2.3).

\subsubsection{Netherlands Railways}

In the following we compare both models on different disruption scenarios from NS. Consequently, we have chosen 12 different scenarios, of which an overview is given in Table 9. The disruptions take place on the main parts of the network, causing one track to be blocked for at least three hours. This track is blocked in both directions, so there is no railway traffic possible there. The parame-

\begin{tabular}{ccccc}
\hline Case & Disruped area & Duration & \\
\hline $1,2,3$ & Gd - Ut & $07: 00-10: 00$ & $16: 00-19: 00$ & $11: 00-15: 00$ \\
4,5 & Rtd - Gv & $16: 00-19: 00$ & $11: 00-15: 00$ & \\
6 & Ledn - Ut & $16: 00-19: 00$ & & \\
7,8 & Amf - Ut & $16: 00-19: 00$ & $11: 00-15: 00$ & \\
9,10 & Gv - Ledn & $16: 00-19: 00$ & $11: 00-15: 00$ & \\
11,12 & Asd - Ut & $16: 00-19: 00$ & $11: 00-15: 00$ & \\
\hline
\end{tabular}

Table 9: Different disruption scenarios NS. The columns show the case numbers, where the disruption is taking place, and on the affected time-slots.

ter settings used for the NS rescheduling instances are given in Table 10. The largest penalty is still on cancelling a trip, this is the most important criterion. The penalties for carriage kilometers and seat-shortage kilometers are the same as in the scheduling case. Three new penalties are used in the rescheduling cases: Shunting Unplanned, Shunting Cancelled, and End Diff. Shunting Unplanned denotes the cost for doing a shunting operation that was not originally scheduled. Every new (un)coupling action requires shunting crew members to perform them. As a consequence, the shunting crew needs to be rescheduled. So, we want to minimize the number of additional shunting movements. On the other hand, Shunting Cancelled stands for the penalty for cancelling a scheduled shunting movement. Shunting crew members are instructed to do planned shunting movements during the day, cancelling such a shunting movement means that the tasks for those crew members have to be cancelled. So, we want to minimize the number of cancelled shunting operations as well. However, cancelling shunting movements is preferred over adding additional shunting movements, because arranging new shunting crew members for a task is usually more diffi- 
cult than cancelling a shunting crew task. Finally, End Diff denotes the penalty for having a negative deviation from the scheduled end of day balance. We leave this coefficient identical to the one used in the planned instance for the start-end-of-day deviation.

\begin{tabular}{lrr}
\hline Coefficient & NS & DSB S-tog \\
\hline Cancel & 1000000 & 1000000 \\
Mileage & 1 & 0.1 \\
Seat Shortage & 0.5 & 0.5 \\
Shunting Unplanned & 1000 & 1000 \\
Shunting Cancelled & 100 & 100 \\
End Diff & 10000 & 10000 \\
\hline
\end{tabular}

Table 10: Penalty values used for the NS and S-tog rescheduling cases. The columns denote the different case numbers, where the disruption in those cases takes place, and on which time-slots.

\subsubsection{DSB S-tog}

For the DSB S-tog instances, there are also 12 different disruption scenarios, see Table 11. These disruptions occur either on the Monday or Friday instances, leading to 24 instances in total.

\begin{tabular}{ccccc}
\hline Case & Disruped Area & \multicolumn{3}{c}{ Duration } \\
\hline $1,2,3$ & Val-Fs & $15: 00-16: 00$ & $15: 00-19: 00$ & $11: 00-12: 00$ \\
$4,5,6$ & Val-Fs & $11: 00-15: 00$ & $09: 00-13: 00$ & $09: 00-10: 00$ \\
$7,8,9$ & Kh-Kk & $15: 00-16: 00$ & $15: 00-19: 00$ & $11: 00-12: 00$ \\
$10,11,12$ & Kh-Kk & $11: 00-15: 00$ & $09: 00-13: 00$ & $09: 00-10: 00$ \\
\hline
\end{tabular}

Table 11: Different disruption scenarios DSB S-tog. The columns denote the different case numbers, where the disruption in those cases takes place, and on which time-slots.

The same objective coefficients are used as in the NS rescheduling instances. The only difference is the mileage penalty, which is 0.1 instead of 1.0, see Section 4.1.3.

\subsubsection{Computational Results}

All three approaches give the same results after rescheduling the disrupted instances. Figure 6 gives an overview of the solution costs for the rescheduling results. Here, the objective value, the mileage costs, and the seat-shortage costs are displayed per rescheduled instance. The other costs are not mentioned, because they are very small in comparison. The objective value is largest in the 
cases where trips are cancelled. In the other cases, the objective value is close to the mileage costs. So, the mileage costs are much larger than the seat-shortage costs in all cases. This can also be noted by Figure 7, where the total seat coverage percentage per rescheduling instance is shown. The seat coverage is always between $97 \%$ and $99.9 \%$, which means that only few passengers do not get a seat in the train.
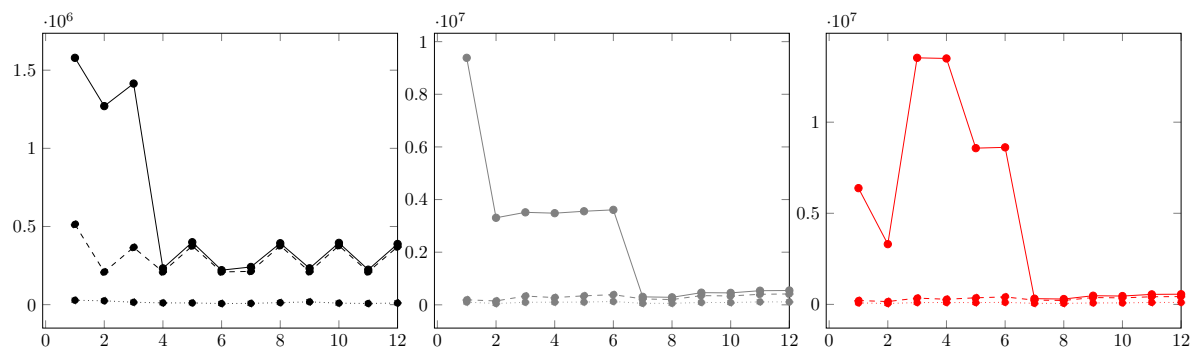

Figure 6: Results for the NS (left) and DSB S-tog (Monday middle, Friday right) rescheduling instances. A straight line denotes the objective value, a dashed line the mileage costs, and a dotted line the seat shortage costs. The horizontal axis denotes the case numbers and the vertical axis the values.

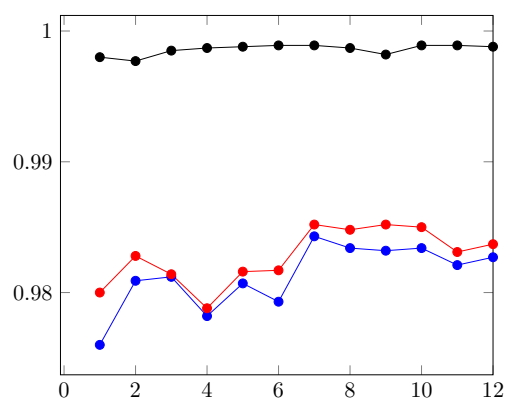

Figure 7: The seat-coverage for the rescheduling cases. The black line is for the Dutch instances, the blue line for the Friday DSB S-Tog instances, and the red line for the Monday DSB S-Tog instances. The horizontal axis denotes the case numbers and the vertical axis the seat cover percentage, where 1 stands for $100 \%$.

Next to finding good results, the computation time is of importance as well. In a disrupted situation, little time is available to reschedule the rolling stock. Therefore, we will compare our models based on their computation times. In Table 12 an overview of all computation times required to solve the different instances with the different models is shown. First of all, the solution method $B$ is substantially faster than the solution method $A$. However, both models 
are considerably slower than the Composition Model. On the other hand, as explained before, the Path Based Model has other advantages over the Composition Model.

We note that the computation times are influenced by the start time of the disruption - a late disruption contains fewer trips than an earlier disruption, thus a late disruption is easier to solve. In conclusion, both models $B$ and $N$ are applicable for usage in practice. They are able to reschedule the rolling stock fast enough such that the rescheduled circulation can be used by practitioners in real-time. On the other hand too high computation times are observed in some cases for the $A$ approach.

\begin{tabular}{|c|c|c|c|c|c|c|c|c|c|}
\hline \multirow[b]{2}{*}{ Case } & \multicolumn{3}{|c|}{ DSBmon } & \multicolumn{3}{|c|}{ DSBfri } & \multicolumn{3}{|c|}{$\mathrm{NS}$} \\
\hline & $A$ & $B$ & $N$ & $A$ & $B$ & $N$ & $A$ & $B$ & $N$ \\
\hline 1 & 38 & 22 & 4 & 18 & 7 & 3 & 722 & 269 & 5 \\
\hline 2 & 4 & 2 & 2 & 5 & 2 & 2 & 20 & 6 & 2 \\
\hline 3 & 72 & 15 & 5 & 113 & 17 & 5 & 138 & 23 & 5 \\
\hline 4 & 63 & 23 & 5 & 51 & 7 & 3 & 11 & 3 & 1 \\
\hline 5 & 176 & 31 & 4 & 151 & 22 & 6 & 133 & 34 & 3 \\
\hline 6 & 286 & 207 & 8 & 303 & 40 & 7 & 14 & 2 & 1 \\
\hline 7 & 8 & 2 & 1 & 13 & 4 & 2 & 13 & 13 & 2 \\
\hline 8 & 6 & 2 & 2 & 9 & 2 & 2 & 159 & 39 & 3 \\
\hline 9 & 62 & 6 & 3 & 96 & 10 & 4 & 10 & 2 & 1 \\
\hline 10 & 106 & 18 & 4 & 103 & 8 & 3 & 157 & 26 & 3 \\
\hline 11 & 222 & 17 & 7 & 284 & 15 & 4 & 15 & 2 & 1 \\
\hline 12 & 156 & 9 & 3 & 235 & 15 & 6 & 163 & 35 & 3 \\
\hline Average & 100 & 29 & 4 & 115 & 12 & 4 & 130 & 38 & 3 \\
\hline
\end{tabular}

Table 12: Computation time (in seconds) for solving the disruption instances.

\subsection{Delayed Transition Constraint Generation}

Finally, in this section we present details of the average computation time and average number of columns and rows of the $A$ and $B$ methods in Table 13 . The results show that the dynamic row generation $B$ method is on average around 5 times faster than the normal column generation method $A$.

Interestingly, the table shows that, on average, the number of generated columns decreases in $B$ for the DSB cases, but increases slightly for the NS cases. However, we observe a consistent decrease in the number of rows.

Although $B$ often results in fewer generated columns and rows, there are cases (considering the non-aggregated results) where this is not the case. There exist cases where runtime is improved even if more columns and rows are generated in total - we believe this is due to the better convergence property of $B$, see Section 3.2 


\begin{tabular}{|c|c|c|c|c|c|c|c|}
\hline \multirow[b]{2}{*}{ Case } & \multicolumn{3}{|c|}{$A$} & \multicolumn{3}{|c|}{$B$} & \multirow{2}{*}{$\begin{array}{r}N \\
\text { Time }\end{array}$} \\
\hline & Time & Columns & Rows & Time & Columns & Rows & \\
\hline DSBfri & 161 & 7040 & 6891 & 16 & 4373 & 4704 & 4 \\
\hline DSBmon & 143 & 7321 & 6523 & 31 & 4701 & 5677 & 4 \\
\hline $\mathrm{NS}$ & 190 & 6178 & 7214 & 51 & 6434 & 3573 & 2 \\
\hline
\end{tabular}

Table 13: The columns show computational time (in seconds), number of columns and rows generated for solving planning and disruption instances. Each row shows the average over 1 planning and 12 disruption cases.

\section{Conclusion}

In this paper a comparison is made between two rolling stock (re)scheduling models by testing them on data sets from two countries (the Netherlands and Denmark). The results show that the considered approaches are not limited or tailored to specific networks. Furthermore, it is the first time that the Composition Model is tested on instances of the DSB S-tog network in Denmark and the Path Based Model on instances from Netherlands Railways.

A sensitivity analysis of the two models is carried out to determine reasonable values for the mileage and seat-shortage penalties for the train services. The results demonstrate that a higher seat-cover requires significantly more kilometers to be carried out by the carriages. For the DSB S-tog case it was, with the different penalties we have tested, not possible to provide a seat for all passengers.

In the current tests the column generation approach of the Path Based Model has longer computation times than the Composition Model. However, we believe that the column generation approach can be quite interesting, especially when we want to include unit specific constraints.

Next to the scheduling instances, we have tested both approaches on rescheduling instances of the networks in the Netherlands and Denmark as well. Disruption instances are smaller than the planned counterparts since all trip decisions up to the start of the disruption are fixed. So in some sense they are easier. However, since we also want to minimize the deviations from the original plan, they are also harder. The experiments show that both models are able to reschedule the rolling circulation fast enough to be used in real-time in both countries.

In future research we want to consider unit specific constraints for the rolling stock, in both a planning and a disruption context. Examples of interesting additional constraints are maintenance appointments and minor rolling stock defects. In the former case units have to reach a maintenance facility in time for an appointment, and in the latter case units with a small defect cannot run on their own but only when coupled to another unit. 


\section{References}

[1] R. K. Ahuja, J. Liu, J. B. Orlin, D. Sharma, and L. A. Shughart. Solving real-life locomotive-scheduling problems. Transportation Science, 39(4): 503-517, 2005.

[2] R.K. Ahuja, T. Magnanti, and J.B. Orlin. Network Flows: Theory, Algorithms and Applications. Prentice-Hall Englewood Cliffs, 1993.

[3] A. Alfieri, R. Groot, L. Kroon, and A. Schrijver. Efficient circulation of railway rolling stock. Transportation Science, 40(3):378-391, 2006.

[4] R. Borndörfer, M. Reuther, T. Schlechte, and S. Weider. A hypergraph model for railway vehicle rotation planning. Proceedings in ATMOS, pages 146-155, 2011.

[5] B. Bouzaiene-Ayari, C. Cheng, S. Das, R. Fiorillo, and W. B. Powell. From single commodity to multiattribute models for locomotive optimization: A comparison of optimal integer programming and approximate dynamic programming. To appear in: Transportation Science, 2014. doi: 10.1287/ trsc.2014.0536.

[6] P. Brucker, J. Hurink, and T. Rolfes. Routing of railway carriages. Journal of Global Optimization, 27(2-3):313-332, 2003.

[7] V. Cacchiani, D. Huisman, M. Kidd, L.G. Kroon, P. Toth, L. Veelenturf, and J.C. Wagenaar. An overview of recovery models and algorithms for realtime railway rescheduling. Transportation Research Part B: Methodological, 63:15-37, 2014.

[8] L. Cadarso and Á. Marín. Robust rolling stock in rapid transit networks. Computers $\& 3$ Operations Research, 38(8):1131 - 1142, 2011.

[9] L. Cadarso and Á. Marín. Improving robustness of rolling stock circulations in rapid transit networks. Computers $\mathcal{E}$ Operations Research, 51:146 - 159, 2014.

[10] J.-F. Cordeau, F. Soumis, and J. Desrosiers. Simultaneous assignment of locomotives and cars to passenger trains. Operations Research, 49(4):531$548,2001$.

[11] J.-F. Cordeau, G. Desaulniers, N. Lingaya, F. Soumis, and J. Desrosiers. Simultaneous locomotive and car assignment at VIA Rail Canada. Transportation Research Part B, 35(8):767-787, 2002.

[12] G. Desaulniers, J. Desrosiers, and M. M Solomon. Column generation, volume 5. Springer, 2005.

[13] P.J. Fioole, L.G. Kroon, G. Maróti, and A. Schrijver. A rolling stock circulation model for combining and splitting of passenger trains. European Journal of Operational Research, 174(2):1281-1297, 2006. 
[14] J.T. Haahr, R.M. Lusby, J. Larsen, and D. Pisinger. A branch-and-price framework for railway rolling stock rescheduling during disruptions. DTU Management engineering, 27p, 2014.

[15] S. Irnich and G. Desaulniers. Shortest path problems with resource constraints. In Column Generation, pages 33-65. Springer US, 2005.

[16] J. Jespersen-Groth, D. Potthoff, J. Clausen, D. Huisman, L. Kroon, G. Maróti, and M. N. Nielsen. Disruption management in passenger railway transportation. Springer, 2009.

[17] L. Kroon, G. Maroti, and L. Nielsen. Rescheduling of railway rolling stock with dynamic passenger flows. Transportation Science, 2014.

[18] N. Lingaya, J.-F. Cordeau, G. Desaulniers, J. Desrosiers, and F. Soumis. Operational car assignment at $\{$ VIA $\}$ rail canada. Transportation Research Part B: Methodological, 36(9):755 - 778, 2002.

[19] L.K. Nielsen, L.G. Kroon, and G. Maróti. A rolling horizon approach for disruption management of railway rolling stock. European Journal of Operational Research, 220(2):496-509, 2012.

[20] M. Peeters and L. Kroon. Circulation of railway rolling stock: a branchand-price approach. Computers $\& 3$ Operations Research, 35(2):538 - 556, 2008 .

[21] S. Rouillon, G. Desaulniers, and F. Soumis. An extended branch-andbound method for locomotive assignment. Transportation Research Part B: Methodological, 40(5):404 - 423, 2006.

[22] B. Vaidyanathan, R. K. Ahuja, and J. B. Orlin. The locomotive routing problem. Transportation Science, 42(4):492-507, 2008.

[23] J.C. Wagenaar, L.G. Kroon, and M. Schmidt. Maintenance in railway rolling stock rescheduling for passenger railways. ERIM report series research in management Erasmus Research Institute of Management, ERS2015-002-LIS, 2015. URL http://hdl .handle.net/1765/77585. 


\begin{tabular}{|c|c|}
\hline \multicolumn{2}{|c|}{ ERIM Report Series Research in Management } \\
\hline ERIM Report Series reference number & ERS-2015-007-LIS \\
\hline Date of publication & $2015-06-29$ \\
\hline Version & $06-07-2015$ \\
\hline Number of pages & 27 \\
\hline Persistent URL for paper & http://hdl.handle.net/1765/78317 \\
\hline Email address corresponding author & jwagenaar@rsm.nl \\
\hline Address & $\begin{array}{l}\text { Erasmus Research Institute of Management } \\
\text { (ERIM) } \\
\text { RSM Erasmus University / Erasmus School } \\
\text { of Economics } \\
\text { Erasmus University Rotterdam } \\
\text { PO Box } 1738 \\
3000 \text { DR Rotterdam, The Netherlands } \\
\text { Phone: +31104081182 } \\
\text { Fax: +31104089640 } \\
\text { Email: info@erim.eur.nl } \\
\text { Internet: http://www.erim.eur.nl }\end{array}$ \\
\hline Availability & $\begin{array}{l}\text { The ERIM Report Series is distributed } \\
\text { through the following platforms: } \\
\text { RePub, the EUR institutional repository } \\
\text { Social Science Research Network (SSRN) } \\
\text { Research Papers in Economics (RePEc) }\end{array}$ \\
\hline Classifications & $\begin{array}{l}\text { The electronic versions of the papers in the } \\
\text { ERIM Report Series contain bibliographic } \\
\text { metadata from the following classification } \\
\text { systems: } \\
\text { Library of Congress Classification (LCC) } \\
\text { Journal of Economic Literature (JEL) } \\
\text { ACM Computing Classification System } \\
\text { Inspec Classification Scheme (ICS) }\end{array}$ \\
\hline
\end{tabular}

